\title{
COURT DECISIONS ON
}

MUSIC PLAGIARISM AND THE PREDICTIVE VALUE OF SIMILARITY ALGORITHMS

Dr. Marc Pendzich Institute of Musicology University of Hamburg

Dr. Daniel Müllensiefen Goldsmiths College University of London 
AGENDA

1 Introduction

2 Method

3 Empirical Study

4 Summary/next steps 
I WIRODUCRION

- Melodic Plagiarism: Huge public interest, importance for pop industry - little research

๑ Exceptions:

- Stan Soocher: They Fought The Law, 1999

- Charles Cronin: Concepts of Melodic Similarity in Music-Copyright, 1998 
I WIRODUCRION

$\odot$ The aim of the study is

- to explore how melodic similarity as measured by modern algorithms is related to court decisions in individual cases

- to measure the similarity of the melody pairs in a sample of cases taken from a collection of court cases and

- to evaluate the predictive power of the algorithmic measurements when compared to the court ruling. 


\section{METIOD}

- 20 cases spanning the years from 1970 to 2005 - with a focus on melodic aspects of music copyright infringement.

- Creation of monophonic MIDI files,

- analysis of the written opinions of the judges,

- reduction of the court decisions to only two categories

(3) "pro plaintiff" = melodic plagiarism

sos "contra plaintiff“ = no infringement 
BRIGUT TUNES VS. MARRIONGS

(1976)

$\odot$ The Chiffons „He‘s So Fine“, 1963

- No. 1 in US, UK highest position 11

๑ George Harrisson, „My Sweet Lord“ Single published in 1971

- No.-1-Hit in US, UK \& (West-)Germany
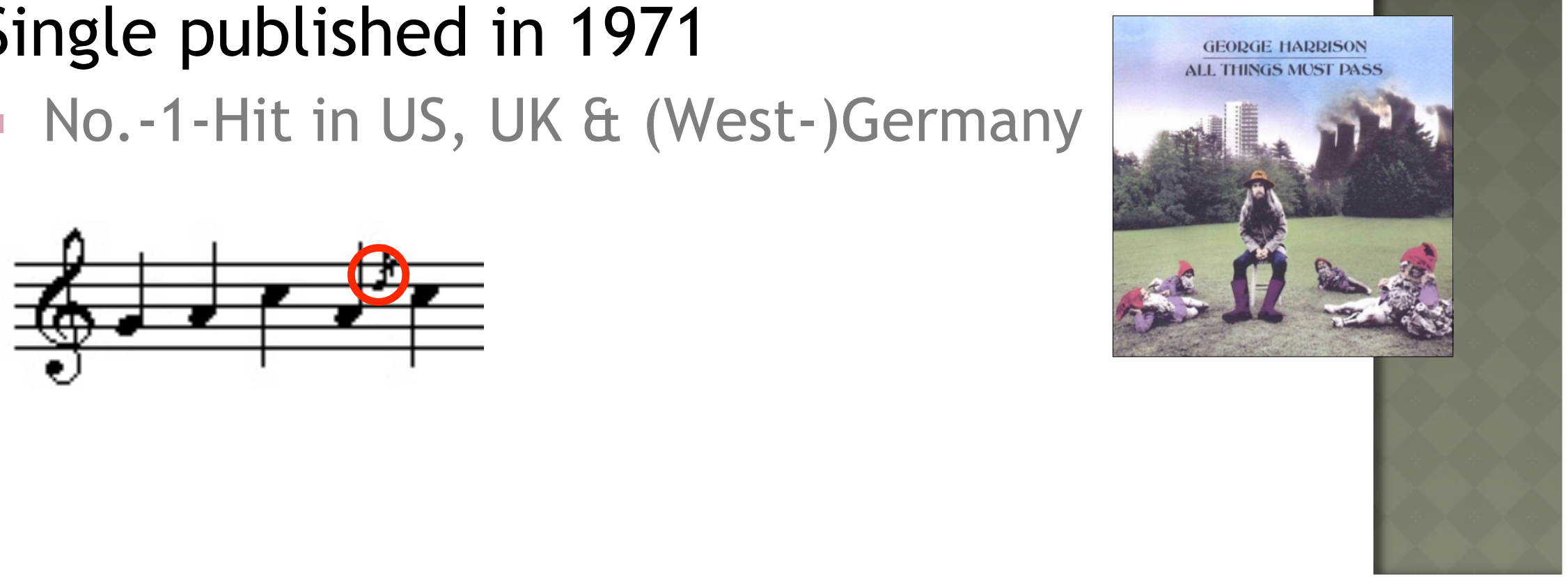
SELLE VS。 GHB (1984)

๑ Ronald Selle, "Let It End"

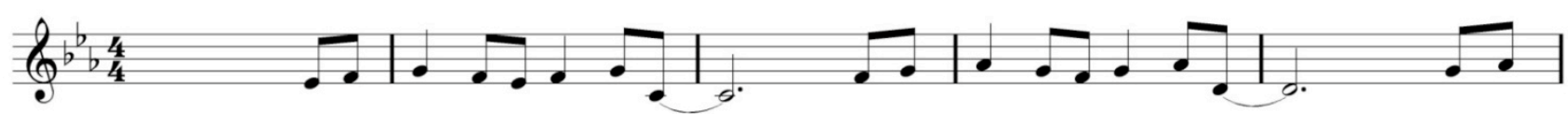

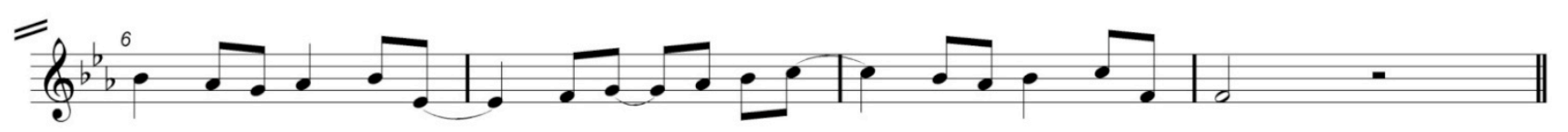

๑ Bee Gees, “How Deep Is Your Love” (1977)

$$
\begin{aligned}
& 6 \text {. }
\end{aligned}
$$

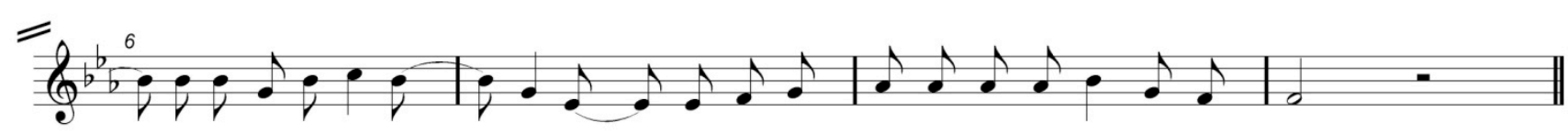


3 EMPURLAL STUDY

- How do court decision relate to melodic similarity?

- What is the frame of reference (directionality of comparisons)?

- How is prior musical knowledge taken into account? 


\section{STAIISTICALLY UNFORMED ALGORUTIMS}

- Idea: Frequency of melodic elements important for similarity assessment

- Inspired from computational linguistics (Baayen, 2001), text retrieval (Manning \& Schütze, 1999)

๑ Conceptual Components:

- m-types (aka n-grams) as melodic elements

- Frequency counts: Type frequency (TF) and Inverted Document Frequency (IDF) 


\section{MELODIC ELEMENTS: M-TYPES}

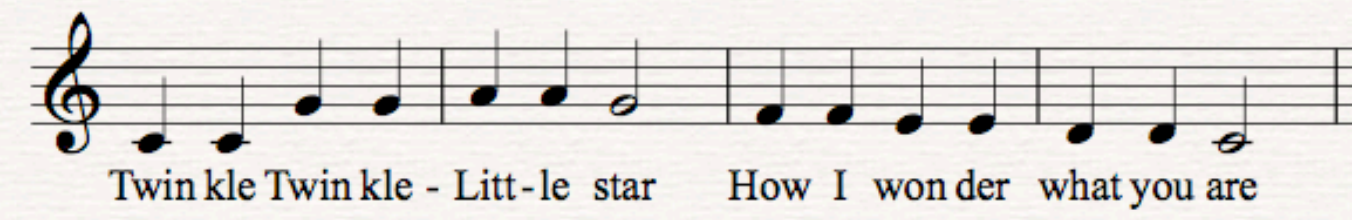

\begin{tabular}{|l|l|l|l|}
\hline Word Type $t$ & Frequency $f(t)$, & $\begin{array}{l}\text { Melodic Type } \tau \text { (pitch } \\
\text { interval, length 2) }\end{array}$ & Frequency $f(\tau)$, \\
\hline Twinkle & 2 & $0,+7$ & 1 \\
\hline little & 1 & $+7,0$ & 1 \\
\hline star & 1 & $0,+2$ & 1 \\
\hline How & 1 & $+2,0$ & 1 \\
\hline I & 1 & $0,-2$ & 3 \\
\hline wonder & 1 & $-2,-2$ & 1 \\
\hline what & 1 & $-2,0$ & 2 \\
\hline you & 1 & $0,-1$ & 1 \\
\hline are & 1 & $-1,0$ & 1 \\
\hline
\end{tabular}




\section{TYPE I INV. DOCUMENT FREQ}

C Corpus of melodies

m melody

$\tau$ Melodic type

$\tau \quad \mathrm{T}$ \# different melodic types

$$
T F(m, \tau)=\frac{f_{m}(\tau)}{\sum_{i=1}^{T} f_{m}\left(\tau_{i}\right)} \quad I D F_{C}(\tau)=\log \left(\frac{|C|}{|m: \tau \in m|}\right)
$$

$|\mathrm{m}: \tau \in \mathrm{m}|$ \# melodies containing $\tau$

\begin{tabular}{|l|l|}
\hline $\begin{array}{l}\text { Melodic Type } \tau \\
\text { (pitch interval, } \\
\text { length 2) }\end{array}$ & Frequency $f(\tau)$ \\
\hline $0,+7$ & 1 \\
\hline$+7,0$ & 1 \\
\hline $0,+2$ & 1 \\
\hline$+2,0$ & 1 \\
\hline $0,-2$ & 3 \\
\hline$-2,-2$ & 1 \\
\hline$-2,0$ & 2 \\
\hline $0,-1$ & 1 \\
\hline$-1,0$ & 1 \\
\hline
\end{tabular}




\section{TYPE I INV. DOCUMENT FREQ}

C Corpus of melodies

m melody

$\tau$ Melodic type

$\tau \quad \mathrm{T}$ \# different melodic types

$$
T F(m, \tau)=\frac{f_{m}(\tau)}{\sum_{i=1}^{\mathrm{T}} f_{m}\left(\tau_{i}\right)} \quad I D F_{C}(\tau)=\log \left(\frac{|C|}{|m: \tau \in m|}\right)
$$

$|\mathrm{m}: \tau \in \mathrm{m}|$ \# melodies containing $\tau$

\begin{tabular}{|l|l|l|}
\hline $\begin{array}{l}\text { Melodic Type } \tau \\
\text { (pitch interval, } \\
\text { length 2) }\end{array}$ & Frequency $f(\tau)$ & $\mathrm{TF}(\mathrm{m}, \tau)$ \\
\hline $0,+7$ & 1 & 0.11 \\
\hline$+7,0$ & 1 & 0.11 \\
\hline $0,+2$ & 1 & 0.11 \\
\hline$+2,0$ & 1 & 0.11 \\
\hline $0,-2$ & 3 & 0.33 \\
\hline$-2,-2$ & 1 & 0.11 \\
\hline$-2,0$ & 2 & 0.22 \\
\hline $0,-1$ & 1 & 0.11 \\
\hline$-1,0$ & 1 & 0.11 \\
\hline
\end{tabular}




\section{TYPE I INV. DOCUMENT FREQ}

C Corpus of melodies

m melody

$\tau$ Melodic type

$\tau \quad \mathrm{T}$ \# different melodic types

$$
T F(m, \tau)=\frac{f_{m}(\tau)}{\sum_{i=1}^{T} f_{m}\left(\tau_{i}\right)} \quad I D F_{C}(\tau)=\log \left(\frac{|C|}{|m: \tau \in m|}\right)
$$

$|\mathrm{m}: \tau \in \mathrm{m}| \quad \#$ melodies containing $\tau$

\begin{tabular}{|l|l|l|l|}
\hline $\begin{array}{l}\text { Melodic Type } \tau \\
\text { (pitch interval, } \\
\text { length 2) }\end{array}$ & Frequency $f(\tau)$ & $\operatorname{TF}(\mathrm{m}, \tau)$ & $\operatorname{IDF}_{\mathrm{c}}(\tau)$ \\
\hline $0,+7$ & 1 & 0.11 & 1.57 \\
\hline$+7,0$ & 1 & 0.11 & 1.36 \\
\hline $0,+2$ & 1 & 0.11 & 0.23 \\
\hline$+2,0$ & 1 & 0.11 & 0.28 \\
\hline $0,-2$ & 3 & 0.33 & 0.16 \\
\hline$-2,-2$ & 1 & 0.11 & 0.19 \\
\hline$-2,0$ & 2 & 0.22 & 0.22 \\
\hline $0,-1$ & 1 & 0.11 & 0.51 \\
\hline$-1,0$ & 1 & 0.11 & 0.74 \\
\hline
\end{tabular}




\section{TYPE I INV. DOCUMENT FREQ}

C Corpus of melodies

m melody

$\tau$ Melodic type

$\tau \quad \mathrm{T}$ \# different melodic types

$$
T F(m, \tau)=\frac{f_{m}(\tau)}{\sum_{i=1}^{T} f_{m}\left(\tau_{i}\right)} \quad I D F_{C}(\tau)=\log \left(\frac{|C|}{|m: \tau \in m|}\right)
$$

$|\mathrm{m}: \tau \in \mathrm{m}| \quad \#$ melodies containing $\tau$

\begin{tabular}{|l|l|l|l|r|}
\hline $\begin{array}{l}\text { Melodic Type } \tau \\
\text { (pitch interval, } \\
\text { length 2) }\end{array}$ & Frequency $f(\tau)$ & $\operatorname{TF}(\mathrm{m}, \tau)$ & $\mathrm{IDF}_{\mathrm{c}}(\tau)$ & TFIDF $_{\mathrm{m}, \mathrm{c}}(\tau)$ \\
\hline $0,+7$ & 1 & 0.11 & 1.57 & 0.1727 \\
\hline$+7,0$ & 1 & 0.11 & 1.36 & 0.1496 \\
\hline $0,+2$ & 1 & 0.11 & 0.23 & 0.0253 \\
\hline$+2,0$ & 1 & 0.11 & 0.28 & 0.0308 \\
\hline $0,-2$ & 3 & 0.33 & 0.16 & 0.0528 \\
\hline$-2,-2$ & 1 & 0.11 & 0.19 & 0.0209 \\
\hline$-2,0$ & 2 & 0.22 & 0.22 & 0.0484 \\
\hline $0,-1$ & 1 & 0.11 & 0.51 & 0.0561 \\
\hline$-1,0$ & 1 & 0.11 & 0.74 & 0.0814 \\
\hline
\end{tabular}




\section{TF=IDF CORRELATION}

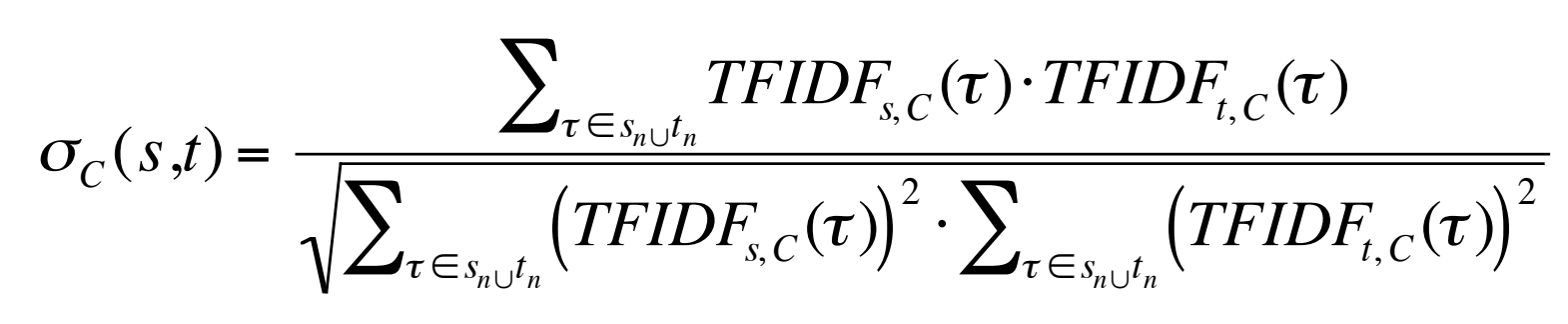




\section{FEATURE=BASED SIMBLARRTY}

Ratio Model (Tversky, 1977): Similarity $\sigma(s, t)$ related to

- \# features in $s$ and $t$ have common

- salience of features $f()$

$$
\sigma(s, t)=\frac{f\left(s_{n} \cap t_{n}\right)}{f\left(s_{n} \cap t_{n}\right)+\alpha f\left(s_{n} \backslash t_{n}\right)+\beta f\left(t_{n} \backslash s_{n}\right)}, \alpha, \beta \geq 0
$$

- features => m-types

- salience $=>$ IDF and TF

- different values of $\alpha, \beta$ to change frame of reference

- Variable m-type lengths $(n=1, \ldots, 4)$, entropy-weighted average 


\section{FEATURE=BASED SIMIARRVY}

Tversky.equal measure (with $\alpha=\beta=1$ )

$$
\sigma(s, t)=\frac{\sum_{\tau \in s_{n}, t_{n}} I D F_{C}(\tau)}{\sum_{\tau \in s_{n} n t_{n}} I D F_{C}(\tau)+\sum_{\tau \in \varepsilon_{s_{n}} \backslash t_{n}} I D F_{C}(\tau)+\sum_{\tau \in \epsilon_{n} \backslash s_{n}} I D F_{C}(\tau)}
$$

Tversky.plaintiff.only measure (with $\alpha=1, \beta=0$ )

$$
\sigma_{\text {plainififfonly }}(s, t)=\frac{\sum_{\tau \in \varepsilon_{s_{n}} h_{n}} I D F_{C}(\tau)}{\sum_{\tau \in s_{n} \cap \lambda_{n}} I D F_{C}(\tau)+\sum_{\tau \in s_{s_{n}} \backslash t_{n}} I D F_{C}(\tau)}
$$

Tversky. defendant. only measure (with $\alpha=0, \beta=1$ )

$$
\sigma_{\text {defendant.only } y}(t, s)=\frac{\sum_{\tau \in \varepsilon_{s_{n}} h_{n}} I D F_{C}(\tau)}{\sum_{\tau \in s_{n} \cap n_{n}} I D F_{C}(\tau)+\sum_{\tau \in t_{n} \backslash s_{n}} I D F_{C}(\tau)}
$$

Tversky.weighted measure with

$$
\alpha=\frac{\sum_{\tau \in s_{n} \cap t_{n}} T F_{s}(\tau)}{\sum_{\tau \in s_{n}} T F_{s}(\tau)} \quad \text { and } \quad \beta=\frac{\sum_{\tau \in s_{n} \cap t_{n}} T F_{t}(\tau)}{\sum_{\tau \in t_{n}} T F_{t}(\tau)}
$$


EVALUATION

$\odot$ Ground Truth: 20 cases with yes/no decision $(7 / 13)$

๑ Evaluation metrics

- Accuracy (\% correct at optimal cut-off on similarity scale)

- AUC (Area Under receiver operating characteristic Curve) 


\section{EVALUATION}

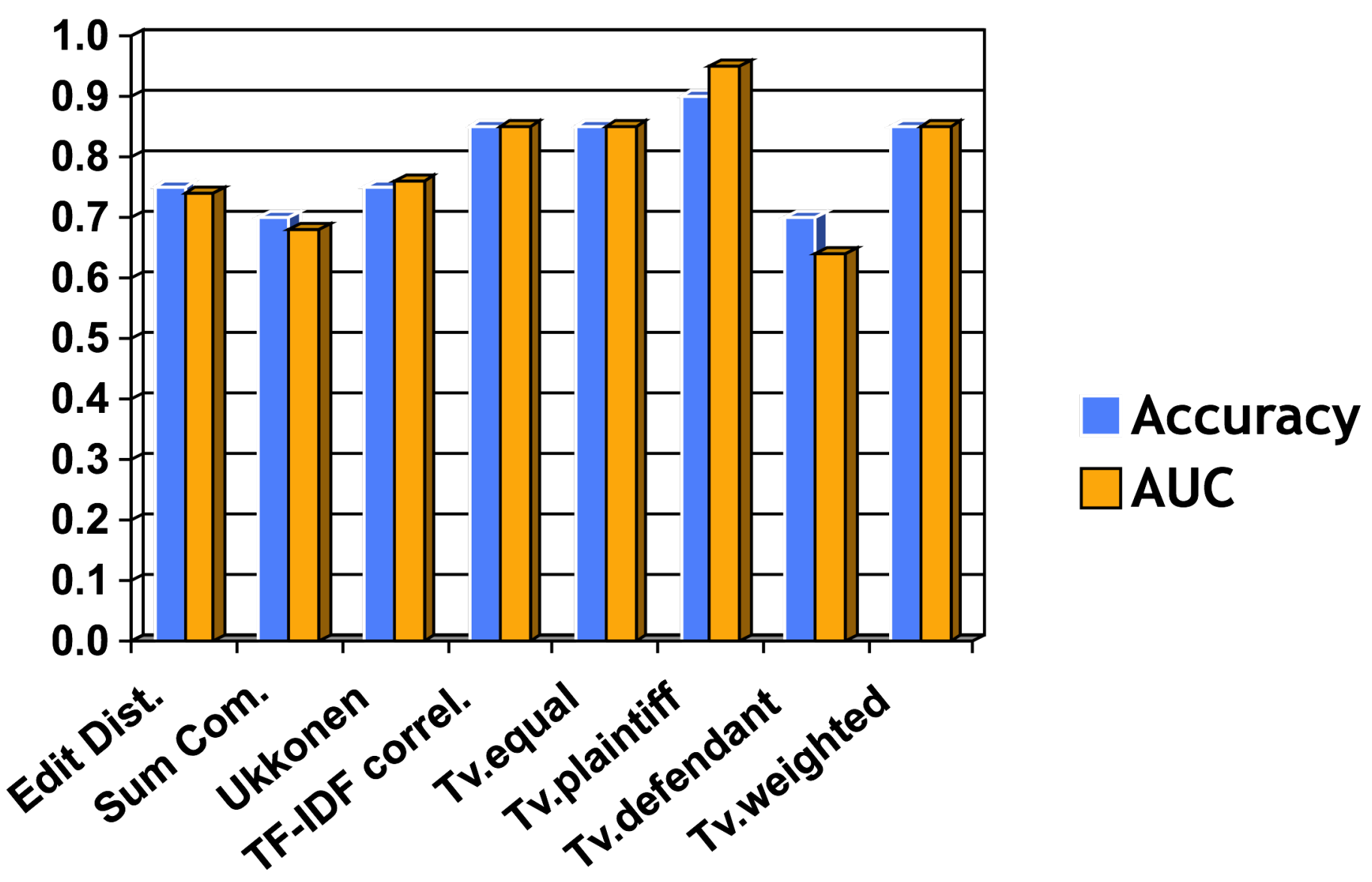




\section{EVALUATUON: ROC CURVES}
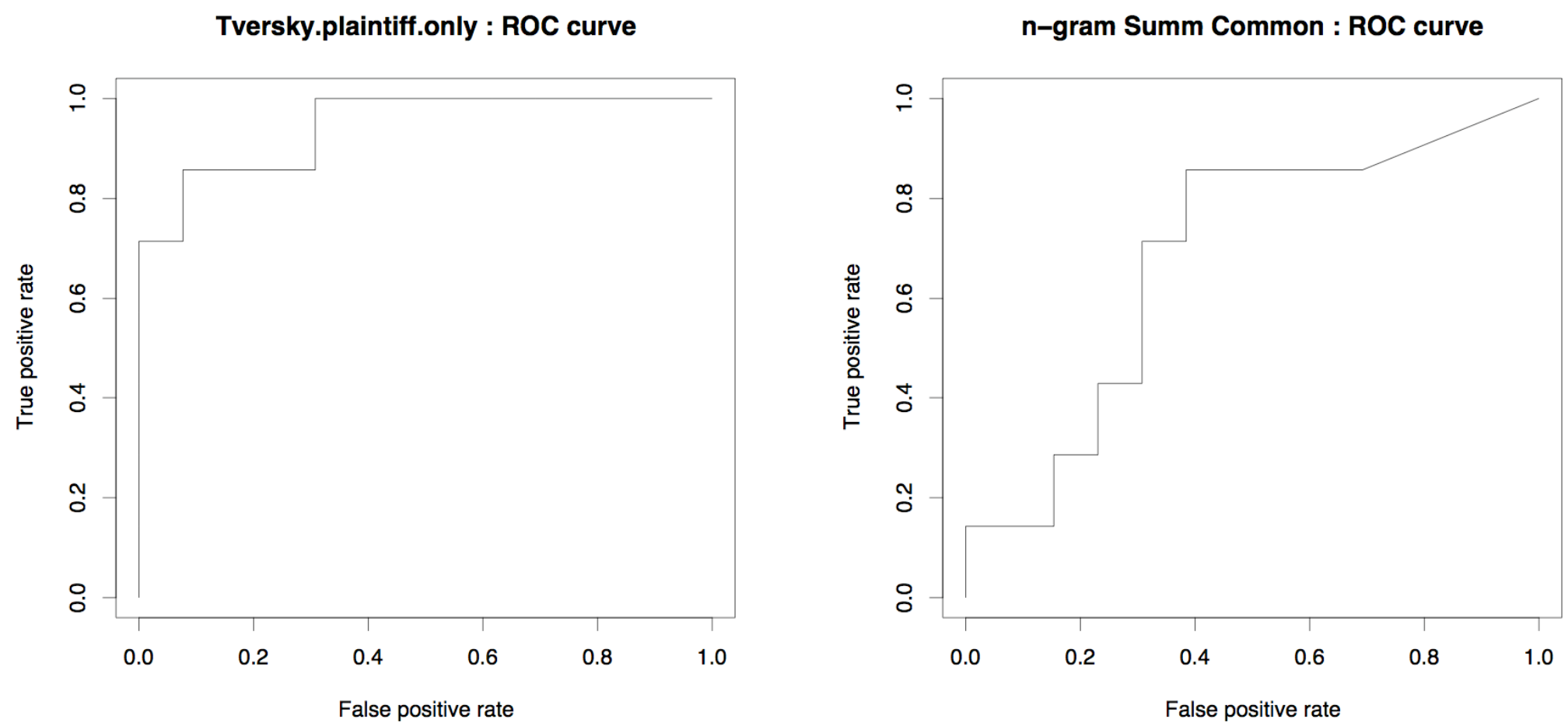


\section{A QUALTVATIVE LOOK}
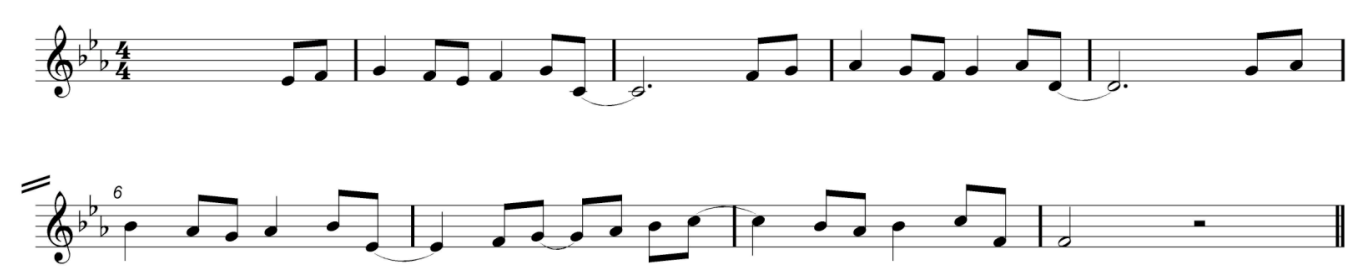

Ronald Selle, “Let It End”

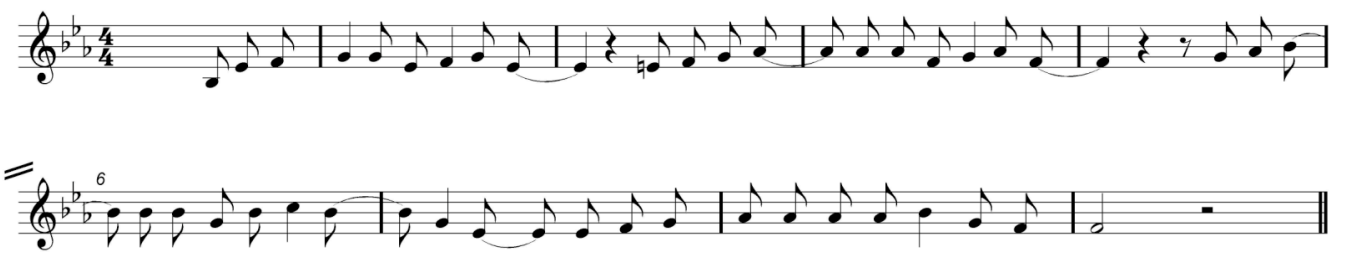

Bee Gees, "How Deep Is Your Love”

\section{Observations:}

- Decision sometimes based on 'characteristic motives'

- High-level form can be important (e.g. call-and-response structure)

- Reference point can be different 
4 SUMMARYANEXT STEPS

- Court decisions can be related closely to melodic similarity

๑ Plaintiff's song is often frame of reference

- Statistical information about commonness of melodic elements is important 
A SUMMARYANEXT STEPS

- More US cases

๑ UK and German cases (from the "big" western markets)

- Include rhythm in m-types

- Compare to more similarity algos from literature 
Thank you for your attention!

\section{COURT DECISIONS ON} MUSIC PLAGIARISM AND THE PREDICTIVE VALUE OF SIMILARITY ALGORITHMS

Dr. Marc Pendzich Institute of Musicology University of Hamburg

Dr. Daniel Müllensiefen Goldsmiths College University of London 